\section{Non-inflammatory IFN}

Type III interferon (IFN- $\lambda$ ) induces signaling cascades and antiviral functions that are very similar to those induced by type I interferon, but signal through a unique IFN- $\lambda$ R1-IL-10R $\beta$ heterodimeric receptor. In Immunity, Galani et al. show that IFN- $\lambda$ is the first interferon produced during influenza virus infection in mice and mediates an antiviral response without inducing inflammation. IFN- $\lambda$ is induced at day 1 post-infection, before, and independently of, type I interferon, and is produced mostly by the respiratory epithelium. IfnIr $1^{-1-}$ mice have increased viral load, neutrophil infiltration, IFN- $\alpha$ production and aggravated pathology in the lung early during infection compared with wild-type or Ifnar $1^{-1-}$ mice. Neutrophils and epithelial cells are the main subsets of cells that express IFN- $\lambda R 1$ and respond to IFN- $\lambda$. In neutrophils, both IFN- $\lambda$ and type I interferon trigger the expression of interferon-stimulated genes and pattern-recognition receptors, whereas only type I interferon triggers the production of proinflammatory cytokines and chemokines. IFN- $\lambda$ treatment early after infection inhibits viral spread and reduces inflammation. IV Immunity 46, 875-890 (2017)

\section{NF- $\kappa B$ citrullination}

Citrullination is a post-translational modification mediated by peptidylarginine deiminases (PADs) that deiminate arginine residues. A greater abundance of citrullinated peptides is associated with joint inflammation in rheumatoid arthritis (RA). In Science Immunology, Sun et al. show that the transcription factor $\mathrm{NF}-\kappa \mathrm{B}$ is modified by arginine citrullination in neutrophils, which express high amounts of PADs. This modification leads to enhanced NF- $\kappa B$ interaction with the nuclear import factor importin- $\alpha 3$ and increased expression of the proinflammatory mediators tumor necrosis factor (TNF) and interleukin $1 \beta$ (IL-1 $\beta$ ). In vitro inhibition of PAD activity in lipopolysaccharide-activated human neutrophils reduces nuclear translocation of the NF- $\kappa B$ p65 (RelA) subunit and attenuates expression of both TNF and IL-1 $\beta$. It remains to be determined whether inhibition of PAD would be beneficial in vivo or would restrict NF- $\kappa \mathrm{B}$-mediated negative feedback and resolution of inflammation.

$L A D$

Sci. Immunol. 2, eaal3062 (2017)

\section{Inducing fetal tolerance}

Antigen-presenting cells (APCs) can prime allogeneic $\mathrm{T}$ cells or induce their tolerance to self-antigens. In Nature, McGovern et al. describe the functional properties of human fetal APCs. They identify dendritic cell (DC) subsets that mirror those found in adult tissues. While fetal DC subsets are capable of responding to Toll-like receptor ligands and priming allogeneic $\mathrm{T}$ cells in vitro, they induce more tolerogenic Foxp $3^{+}$regulatory $\mathrm{T}$ cells than adult DCs do. T cells cocultured with fetal DCs likewise proliferate less and produce fewer proinflammatory cytokines than T cells cultured with adult DCs.

Fetal DCs express high amounts of arginase-2, which suppresses the expression of tumor necrosis factor. Thus fetal DCs are programmed to induce tolerogenic responses to allogeneic antigens encountered in utero.

$L A D$

Nature (14 June 2017) doi:10.1038/nature22795

\section{Alzheimer's disease microglia}

It remains unclear whether microglial function is detrimental or protective but insufficient during neurodegenerative diseases. In Cell, Amit and colleagues describe their use of massive parallel single-cell RNA-seq in a mouse model that expresses five Alzheimer's disease (AD)-associated mutations (5XFAD mice) to identify a distinct microglia subset present in $\mathrm{AD}$, but not in healthy mice. These $\mathrm{AD}$-associated microglia downregulate the expression of homeostatic genes $(\mathrm{C} \times 3 \mathrm{Cr} 1)$ and upregulate the expression of $\mathrm{AD}$ risk factors (Apoe, Trem2) and genes associated with lysosomal-phagocytic and lipid metabolism pathways as the disease progresses. These cells can be identified on the basis of the expression of CD9, CD11c, Clec7a and CD63, and are localized in the vicinity of $\beta$-amyloid plaques in 5XFAD mice and in brain samples from people with $\mathrm{AD}$. Fully differentiated $\mathrm{AD}$-associated microglia are absent in Trem $2^{-/-}$mice, which have accelerated AD compared with wild-type mice on the 5XFAD background, thus suggesting that $\mathrm{AD}$-associated microglia might be protective.

Cell (8 June 2017) doi:10.1016/j.cell.2017.05.018

\section{MAIT cell heterogeneity}

Mucosa-associated invariant T cells (MAIT cells) recognize riboflavin metabolites displayed via the antigen-presenting molecule MR1. In the Proceedings of the National Academy of Sciences, Sandberg and colleagues investigate potential heterogeneity in the response of human MAIT cells to either Escherichia coli or Candida albicans. Although both of these microbes possess riboflavin biosynthesis pathways, they elicit distinct responses from MAIT cells, with the response to $E$. coli being generally more robust and polyfunctional with greater $T$ cell receptor (TCR) downregulation. The TCR V $\beta$ usage also influences the strength and tone of the MAIT cell response, which is suggestive of the recognition of distinct ligands. Analysis of the MAIT cell surface proteome and transcription factors reinforced their distinction from other T cell subsets. The findings also highlight a degree of functional heterogeneity, with MAIT cells that express the markers CD56, CD84 and CD94 having more potent responses to stimulation by IL-12 plus IL-18.

Proc. Natl. Acad. Sci. USA (19 June 2017) doi:10.1073/ pnas. 1705759114

\section{Breaking down barriers}

Tumors are often encapsulated by extracellular matrix (ECM), which can act as a barrier to entry by immune cells. In The Journal of Clinical Investigation, Smyth and colleagues investigate the role of heparanasean enzyme involved in degrading the ECM-in controlling the entry of natural killer (NK) cells into the tumor mass. Resting NK cells express only low levels of heparanase, but amounts of the active version of the enzyme increase dramatically after cell stimulation. Conditional knockout of heparanase in NK cells has no effect on their development, function, proliferation or distribution. However, in several different models, mice with heparanase-deficient NK cells have an impaired ability to control tumors but show increased frequency of exclusion from the tumor mass. The expression of heparanase by NK cells is therefore important for their invasion of tumors, probably owing to degradation of the ECM capsule.

J. Clin. Invest. (21 June 2017) doi:10.1172/JCI92958 